\title{
Microvascular Development in Porcine Right and Left Ventricular Walls
}

\author{
HIDEO OHUCHI, PATRICIA E. BEIGHLEY, YUE DONG, MAIR ZAMIR, AND ERIK L. RITMAN
}

\begin{abstract}
Department of Pediatrics [H.O.], National Cardiovascular Center, Fujishiro-dai, Suita 565-8565, Osaka, Japan; Department of Physiology and Biomedical Engineering [P.E.B., Y.D., E.L.R.], Mayo Clinic College of Medicine, Rochester, Minnesota 55905; Departments of Applied Mathematics and of Medical Biophysics [M.Z.], University of Western Ontario, London, Ontario, N6A 5B7, Canada
\end{abstract}

\begin{abstract}
Patients with congenital heart disease who have a morphological right ventricle (RV) serving as a systemic ventricle have an increased incidence of RV dysfunction. A different structural response of microvessels to increased pressure load in the RV is a possible mechanism for this dysfunction. To examine the merit of this hypothesis, we explored the possibility that in the normal heart, the branching architecture of microvasculature in walls of the left ventricle (LV) and RV mature differently. The branching structure of intramyocardial arterioles and their downstream branches were investigated using three-dimensional (3D) micro-computed tomography (CT) images in different regions of the RV and LV walls of normal fetal, 1-mo, and 5-mo old pigs. The results point to a significant difference in the volume of myocardium perfused per vessel cross-sectional area (CSA) between the LV and RV walls at 5 mo. We speculate that this difference may be related to the reserve functional capacity of the LV, which requires a corresponding reserve in the expansion capacity of vasculature in the LV wall. (Pediatr Res 61: 676-680, 2007)
\end{abstract}

$\mathrm{S}$ ome patients with congenital heart disease, such as those with transposition of the great arteries after an atrial surgical correction, have a RV serving as a systemic ventricle. Some of these patients subsequently show a gradual deterioration of RV function (1). Recent study of such patients has shown that abnormal RV function is associated with impaired myocardial perfusion and that this, in turn, is related to a delay of the surgical correction (2). In addition, these patients exhibit reduced coronary flow reserve (3). Conversely, longterm preserved systemic RV function has been reported in some patients after corrective surgery (4), implying that factors associated with early microvascular development may have some impact on impaired RV adaptation to chronic systemic pressure. Recently, Kassab et al. (5) reported that in 3- to 4-mo-old pigs, no major structural differences in branching architecture of the three major coronary arteries were observed. However, because the pigs in that study were relatively mature, the growth-related branching structure of the coronary arteries remains unknown. In sheep, it has been found that myocardial blood flow dramatically changes from

Received September 19, 2006; accepted January 24, 2007.

Correspondence: Erik L. Ritman, M.D., Ph.D., Department Physiology and Biomedical Engineering, Alfred 2-409, Mayo Clinic College of Medicine, 200 First Street SW, Rochester, MN 55905; e-mail: elran@mayo.edu

This study was supported by NIH research grant EB000305, National Institutes of Health, Bethesda, MD.

DOI: $10.1203 /$ pdr.0b013e31805365a6 the fetus, newborn through adult and that these changes are related to change in work load of both ventricles (6). Based on this background, we hypothesize that the microvascular bed of the RV in the mature animal reduces its ability to respond to the myocardial hypertrophy induced by exposure to systemic pressure late in the maturation phase of the heart and hence results in a gradual deterioration of RV function.

\section{METHODS}

Specimen acquisition. All animal studies were approved by the Mayo Foundation's Institutional Animal Care and Use Committee. The study population consisted of three fetuses (gestational age, $98 \mathrm{~d}$ ), three 1-mo-old and four 5-mo-old pigs. All animals were euthanized with a weight-adjusted intravenous (i.v.) injection of Sleepaway (Fort Dodge Laboratories, Fort Dodge, IA). The average body weight at this time was $0.4 \pm 0.02,10 \pm 1$, and $62 \pm 2 \mathrm{~kg}$ in the fetus, 1-mo-old, and 5-mo-old pigs, respectively. After the heart was harvested, the two coronary ostia were cannulated with a 2-mm diameter plastic cannula. For the fetal heart, the cannula was placed in the ascending aorta. To clear the coronary network of blood, $60 \mathrm{~mL}$ of heparinized saline was infused $(0.9 \%$ sodium chloride with $10,000 \mathrm{U} / \mathrm{L}$ heparin) in each coronary artery. Then a liquid, low-viscosity polymer containing $\mathrm{PbCrO}_{4}$ as an opacifying agent (Microfil ${ }^{\circledR}$ MV-122; Flow Tech, Inc., Carver, MA) was infused at $100 \mathrm{~mm} \mathrm{Hg}$ for the 1-mo-old and 5-mo-old pig hearts and at $60 \mathrm{~mm}$ $\mathrm{Hg}$ for the fetal hearts, and this infusion was continued until the compound flowed freely from the coronary sinus. The hearts were then immersed in $10 \%$ neutral buffered formalin and refrigerated at $4^{\circ} \mathrm{C}$ overnight to allow polymerization of the compound. As illustrated at the top of Figure 1, five transmural specimens about $2.0 \mathrm{~cm}$ long and $1.0 \mathrm{~cm}$ wide, three from the RV wall (inlet, free wall, and outlet) (7) and two from the LV (free wall and outflow), were removed from each of the 1-mo-old and 5-mo-old pig hearts for micro-CT scanning. The fetal hearts were scanned intact. The specimens were then placed in a $75 \%$ alcohol solution. At 15-min intervals, the alcohol concentration was increased to $85 \%, 95 \%$, and finally to $100 \%$ to progressively dehydrate the tissue. The specimens were then immersed in low melting point paraffin wax for subsequent scanning and 3D image reconstruction. The number of animals used and the number of specimens from each animal are summarized in Table 1.

$3 D$ micro-CT reconstruction. Specimens were scanned intact by a micro-CT system described elsewhere (8). For this study, the micro-CT scanner was configured such that the side dimension of the cubic voxels was $20 \mu \mathrm{m}$ (16-bit gray scale). The resulting 3D images were displayed and analyzed using image analysis software (Analyze ${ }^{\circledR}$ 6.0, Biomedical Imaging Resource, Mayo Clinic, Rochester, MN).

CT image analysis. Each voxel in a 3D micro-CT image has its own gray-scale value related to the $\mathrm{x}$-ray attenuation of the tissue in that location. Bright values indicate the contrast agent in the vessel lumen, and low gray-scale values indicate muscle and other soft tissue. To separate vessels from tissue, the gray-scale value of a myocardial area without obvious vessels and of the center of a large contrast-filled vessel lumen were first determined. Next, we determined a minimum gray-scale (i.e. segmentation threshold) for all vessels in the specimen by adding $20 \%$ of the difference between the

Abbreviations: CSA, cross-sectional area; LV, left ventricle; PV, perfused volume; $\mathbf{R V}$, right ventricle 
RV
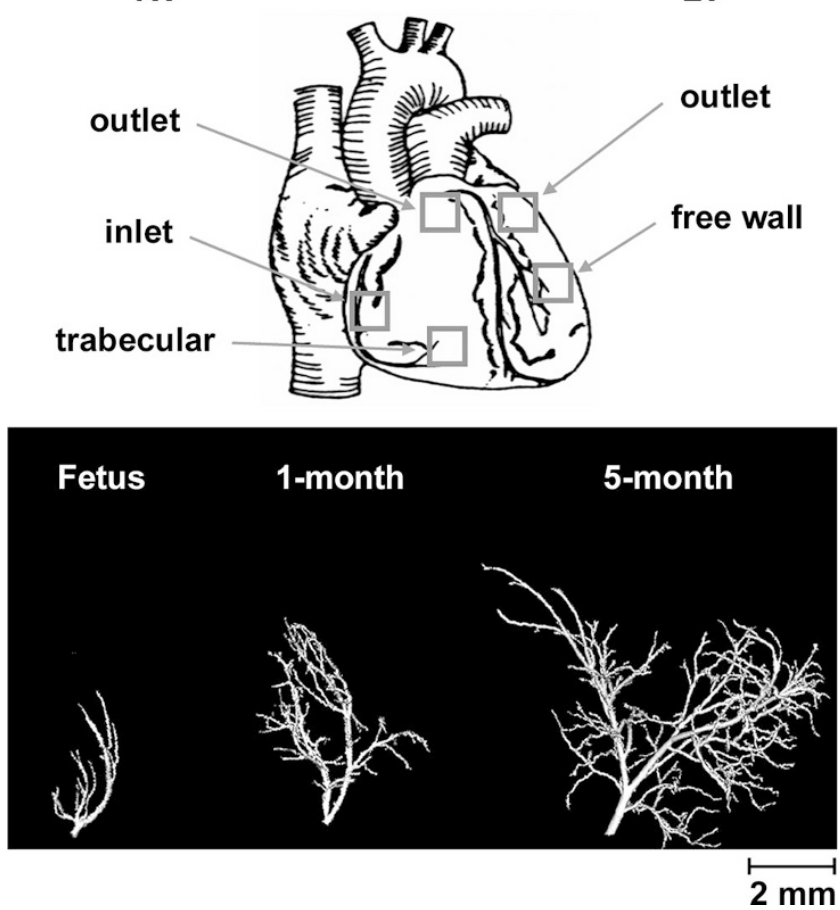

Figure 1. (Top) Location of myocardial "biopsies" from the RV and LV walls. (Bottom) Micro-CT images of RV inflow myocardial arterioles with their distal branches digitally isolated from their 3D micro-CT images. The endocardium is at the upper edge and the epicardium is at the lower edge of the panel. The image from the fetus is on the left, the image from 1-mo-old is in the middle, and the image from the 5-mo-old pig is on the right.

gray-scales of tissue and within a large vessel lumen to the gray-scale of the nonopacified myocardium.

Quantitative morphometric analysis of intramyocardial arteriolar trees. Intramyocardial arterioles of approximately 180 to $200 \mu \mathrm{m}$ in diameter were identified and isolated. Representative arterioles for each age group, with their distal branches and sub branches in the RV inlet wall, are shown at the bottom of Figure 1. Each arteriolar tree was then analyzed to provide values of interbranch segment lumen, diameter, length, and total lumen volume, as illustrated in Figure 2. The root segment of the main arteriole was considered as being at level 0 (generation 0 ), its two branches were then considered to be at level 1 (generation 1), and their branches as being at level 2 (generation 2), etc. The $j, k$ notation of Zamir (9) was used to place these segments within the full tree structure of the selected arteriolar tree.

The cumulative length $\left(\mathrm{L}_{2}\right)$ of a given vascular path was defined as the sum of the lengths of the vessel segments that make up that path as illustrated in Figure $2 B(10)$. The length of the straight line joining the two ends of the path was then designated as $\mathrm{L}_{1}$, and an index of tortuosity of that path was defined as the ratio $\mathrm{L}_{2} / \mathrm{L}_{1}$.

In addition to the lumen diameters of the parent and its larger and smaller daughter branches $\left(d_{0}, d_{1}, d_{2}\right)$, the angles that the two branches make with the direction of the parent branch $\left(\theta_{1}\right.$ and $\left.\theta_{2}\right)$ were also measured (Fig. $2 C$ ). The optimum angles that the two branches make with the direction of the parent vessel have been determined theoretically to depend on the bifurcation index $\alpha\left(=\mathrm{d}_{2} / \mathrm{d}_{1}\right)(9)$ and under the idealized conditions of Murray's law (11) whereby the three diameters at a bifurcation are related by:

$$
\left(d_{0}\right)^{3}=\left(d_{1}\right)^{3}+\left(d_{2}\right)^{3}
$$

Blood flow in a vessel segment is driven by a pressure decrease from upstream to the downstream end of the vessel. Under the idealized conditions of Poiseuille flow, this pressure decrease is related to the diameter (d) and length (1) of the vessel and to the flow rate through it as well as the viscosity of the fluid (12). In a vascular tree, as the flow proceeds along a certain path from one level of the tree to the next, the total pressure drop $(\Delta \mathrm{P})$ along that path is the sum of pressure drops along the vessel segments comprising that path. Based on Murray's law (see equation 1), $\Delta \mathrm{P}$ along the largest path within the tree, normalized in terms of the absolute value of the pressure drop $\Delta \mathrm{P}_{0}$ along the first segment within the tree is given by (13):

$$
\frac{\Delta P}{\left|\Delta P_{0}\right|}=-1-\left(\frac{l_{1} / d_{1}}{l_{0} / d_{0}}\right)-\left(\frac{l_{2} / d_{2}}{l_{0} / d_{0}}\right)-\ldots-\left(\frac{l_{N} / d_{N}}{l_{0} / d_{0}}\right),
$$

\begin{tabular}{|c|c|c|c|c|c|}
\hline \multirow{3}{*}{$\begin{array}{c}\text { BW, kg } \\
\text { Ventricle } \\
\text { Specimen location }\end{array}$} & \multicolumn{5}{|c|}{$\begin{array}{c}\text { Fetus }(n=3) \\
0.4 \pm 0.02\end{array}$} \\
\hline & \multicolumn{3}{|c|}{$\mathrm{RV}$} & \multicolumn{2}{|c|}{ LV } \\
\hline & Inflow & Free wall & Outflow & Free wall & Outflow \\
\hline No. of specimens & 3 & 3 & 3 & 3 & 3 \\
\hline Wall thickness, mm & $4 \pm 1$ & $4 \pm 1$ & $3 \pm 1$ & $5 \pm 1$ & $5 \pm 1$ \\
\hline $\begin{array}{l}\text { No. of arterioles } \\
\text { analyzed }\end{array}$ & 3 & 6 & 15 & 8 & 7 \\
\hline \multirow[t]{4}{*}{ Root diameter, $\mu \mathrm{m}$} & $190 \pm 10$ & $192 \pm 9$ & $190 \pm 8$ & $188 \pm 7$ & $189 \pm 8$ \\
\hline & \multicolumn{5}{|c|}{$\begin{array}{c}1 \mathrm{mo}(n=4) \\
9.0 \pm 1.0\end{array}$} \\
\hline & \multicolumn{3}{|c|}{$\mathrm{RV}$} & \multicolumn{2}{|c|}{ LV } \\
\hline & Inflow & Free wall & Outflow & Free wall & Outflow \\
\hline No. of specimens & 3 & 3 & 4 & 3 & 2 \\
\hline Wall thickness, mm & $7 \pm 1$ & $4 \pm 2$ & $4 \pm 1$ & $11 \pm 2$ & 13 \\
\hline $\begin{array}{l}\text { No. of arterioles } \\
\text { analyzed }\end{array}$ & 9 & 9 & 8 & 12 & 15 \\
\hline \multirow[t]{4}{*}{ Root diameter, $\mu \mathrm{m}$} & $191 \pm 7$ & $191 \pm 8$ & $189 \pm 9$ & $190 \pm 8$ & $191 \pm 9$ \\
\hline & \multicolumn{5}{|c|}{$\begin{array}{c}5 \mathrm{mo}(n=3) \\
62.0 \pm 1.0\end{array}$} \\
\hline & \multicolumn{3}{|c|}{ RV } & \multicolumn{2}{|c|}{ LV } \\
\hline & Inflow & Free wall & Outflow & Free wall & Outflow \\
\hline No. of specimens & 3 & 3 & 3 & 3 & 3 \\
\hline Wall thickness, mm & $12 \pm 1$ & $7 \pm 1$ & $10 \pm 3$ & $23 \pm 2$ & 22 \\
\hline No. of arterioles & 10 & 5 & 10 & 4 & 5 \\
\hline
\end{tabular}

Table 1. Characteristics of specimens

analyzed

Root diameter, $\mu \mathrm{m} \quad 189 \pm 8 \quad 192 \pm 8 \quad 191 \pm 9 \quad 192 \pm 8 \quad 189 \pm 10$

Values are mean $\pm \mathrm{SD}$. BW, body weight; RV, right ventricle; $\mathrm{LV}$, left ventricle.

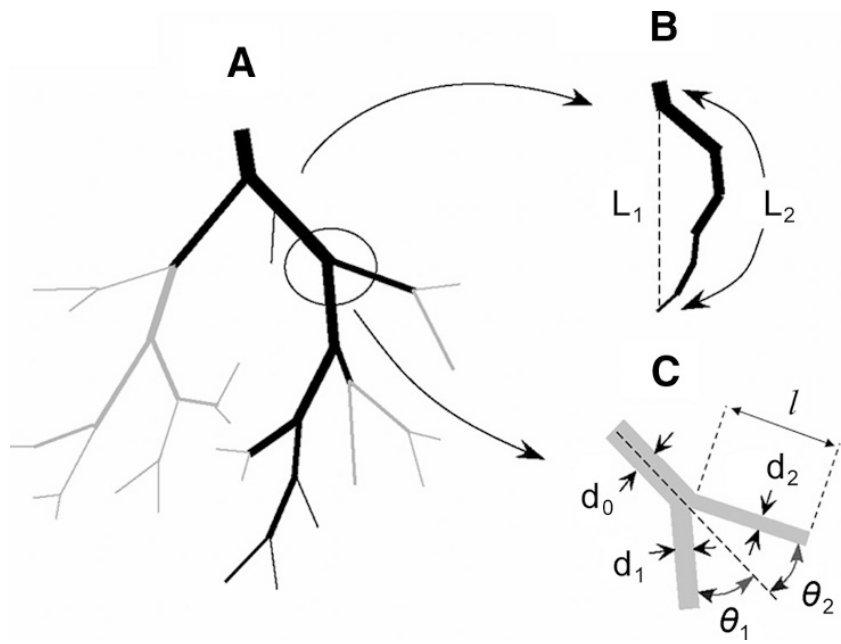

Figure 2. Tree analysis. (A) The main arteriole (black) of an arteriolar tree is defined as the one that has the largest number of branch points. $(B)$ Tortuosity is defined as the ratio $\mathrm{L}_{2} / \mathrm{L}_{1}$. $(C) \mathrm{d}_{0}, \mathrm{~d}_{1}$, and $\mathrm{d}_{2}$ are diameters of parent, larger, and smaller daughter branches, respectively, and $\theta_{1}$ and $\theta_{2}$ are angles that the two daughters make, as shown, and $l$ is the length of a vessel segment.

where $\mathrm{N}$ is the number of vessel segments along the selected path. All the quantities on the right side of this equation are available from our 3D micro-CT images so that the pressure decrease on the left can be evaluated. 
A

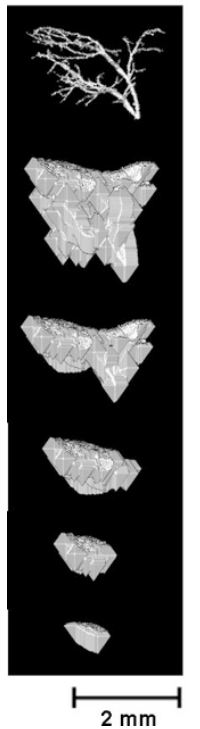

B

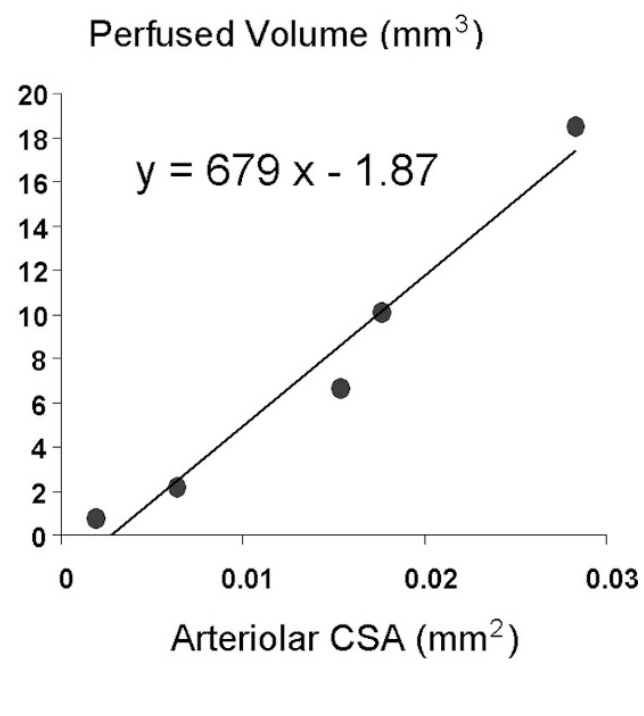

Figure 3. The 3D image of an isolated arteriolar tree ( $A$, top) is broken down digitally into progressively distal trees by the process of "pruning," as described in the text, and then each pruned tree is "homotopically dilated," as described in the text. (A) All the dilated, "pruned," trees are shown. The volume of added voxels is decreed to be the volume of myocardial tissue perfused by the selected arteriole. $(B)$ The relationship between the perfused myocardial volume and CSA of the perfusing arteriole.

The total lumen volume of the arteriolar tree was calculated by summing the volume of all the voxels within the image opacified arterial tree. This process was then repeated for each subtree obtained by "pruning" at each junction along the main path. We also calculated the volume of myocardium perfused by that arterial tree and each of these subtrees. For the purpose of this calculation, the binary image of the selected tree was "dilated" by adding voxels to its surface to produce a dilated volume. The rate of increase in the dilated volume was calculated and plotted against the number of voxels added to the vessels' lumens. Initially, this increased in proportion to the surface area of the lumen of the tree. Ultimately, however, a point was reached beyond which the rate of volume increase was markedly reduced. This occurs when the spaces between branches were filled, so that only the outer surface of the dilated volume is subject to further addition of voxels. At this point, the process was terminated. The perfused volume (PV) of tissue by the arteriole was then calculated by simply subtracting the total volume of the arteriolar tree from the final dilated volume. Each pruned branch was dilated to estimate its $\mathrm{PV}$, and all the PVs were plotted against the corresponding branch's lumenal CSA, as shown in Figure 3 (RV inflow region in a 1-mo-old pig). Slopes of regression lines between the CSA and downstream lumen volume and between CSA and PV were calculated.

Statistical analysis. All data are presented as mean \pm SD or SE, where appropriate. One-way analysis of variance (ANOVA), followed by Bonferroni's post hoc test with correction for multiple comparisons was used to identify the statistical differences among the three age groups. A repeatedmeasures ANOVA with group and generation interaction was used for the serial measurements of CSA, the pressure decrease along the main path and angle between main and branch paths. Correlation between the continuous variables was analyzed using linear regression analysis. Individual group

Lumen Volume/CSA $\left(\mathrm{mm}^{3} / \mathrm{mm}^{2}\right)$

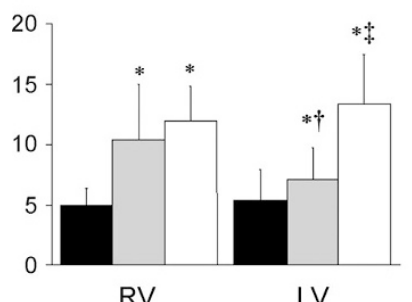

Figure 5. Lumen volume (left) and PV (right) per CSA among the age-based groups. Fetal pig ( $n=9 \mathrm{RV}, n=6 \mathrm{LV}$; solid columns), 1-mo-old pig $(n=$ $10 \mathrm{RV}, n=5 \mathrm{LV}$; shaded columns) and 5-mo-old pig ( $n=9 \mathrm{RV}, 6 \mathrm{LV}$; open columns). At $5 \mathrm{mo}$, the ratio of RV and LV lumen volume/CSA are comparable, but the ratio of PV/CSA in LV is significantly higher than that in RV. $*$ * Statistical significance versus fetal and 1-mo-old pig heart, respectively. $\dagger$ Statistical significance versus $\mathrm{RV}$. Values are mean $\pm \mathrm{SD}$.

comparisons were performed by an unpaired student $t$-test. A value of $p<$ 0.05 was considered significant in all analyses.

\section{RESULTS}

The results in Figure 4 show that as the vascular trees of the ventricular walls develop with age, the arteriolar lengths and number of generations increase, with the latter reaching a plateau in the RV wall at as early as $1 \mathrm{mo}$. As the Figure 4 shows, there was no difference in the tortuosity among the three age-based groups or between the RV and LV, except for the slightly higher tortuosity in the fetal RV wall (statistically significant, with $p<0.05$ ). There was no statistically significant difference among the five region-based groups in the number of generations reached in each age group.

The results in Figure 5, left show that the total vascular lumen volume/CSA increases with age. The PV per unit CSA also increases with age, more so in the LV than in the RV (Fig. 5 , right). The ratio of PV/CSA is significantly higher in the 5-mo-old LV than in the same age RV.

The results in Figure 6 compare the sum of the cubes of the two branch radii with the cube of the parent radius at vascular junctions (left) and the corresponding branching angles at vascular junctions (right), showing very little difference among the age-based groups or between all the locations in the $\mathrm{RV}$ and LV walls in each group. The results suggest that the basic 3D structure is established before birth.

The results in Figure 7 show the cumulative pressure decrease along vascular paths within the LV and RV walls and for the three age groups, indicating no major difference between pressure gradients in the microvascular beds of the RV and LV walls.
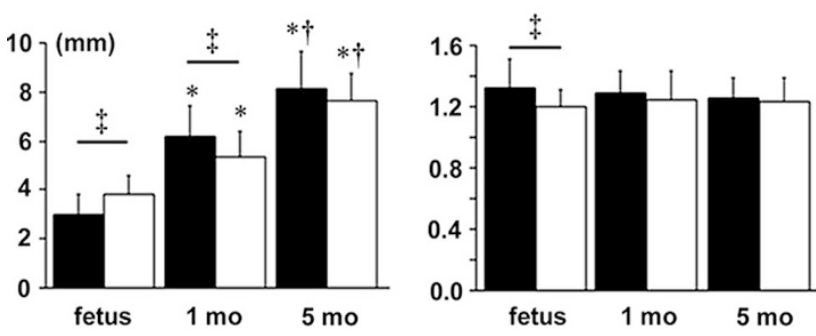

Figure 4. Respectively from left to right, comparison of main arteriolar length, tortuosity, and generations among the age-based groups. RV (solid columns) and LV (open columns). $n$ values for RV and LV are 9 and 6 for the fetal pig, 10 and 5 for the 1-mo-old pig, and 9 and 6 for the 5-mo-old pig. * + Statistical significance versus fetal and 1-moold pig heart, respectively. $\ddagger$ Significance between RV and LV. Values are mean $\pm \mathrm{SD}$. 
Sum of cubes of daughter radii $\left(\times 10^{-3}\right)$

Total branching angle $\left(\theta_{1}+\theta_{2}\right)$

fetus
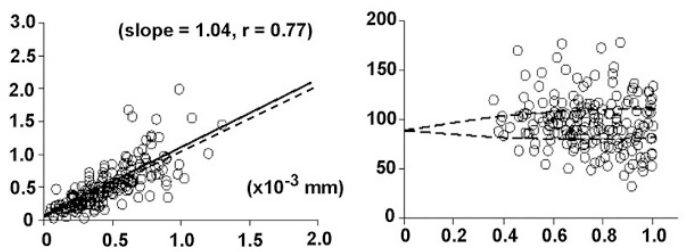

$1 \mathrm{mo}$
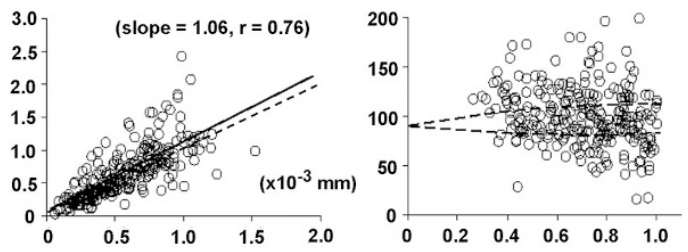

$5 \mathrm{mo}$
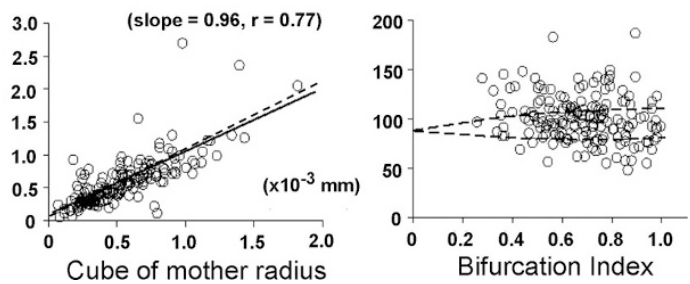

Figure 6. (Left) Relationship between the cube of the parent radius and the sum of its two daughters' radii cubed in fetal pig, 1-mo-old pig, and 5-mo-old pig in all regions of the RV and LV. Dashed diagonal line indicates theoretical "cube law" relationship (9). (Right) Relationship between the bifurcation index and total branching angles $\left(\theta_{1}+\theta_{2}\right)$ in fetal pig, 1-mo-old pig, and 5-mo-old pig in all regions of the RV and LV. Dashed lines represent optimal relationship based on the theoretical consideration (9). Data represents all myocardial regions in both ventricles.

\section{DISCUSSION AND CONCLUSIONS}

Micro-CT image and study limitations. An advantage of micro-CT is that a relatively large intact volume of opaque tissue can be analyzed with 3D high-resolution (8). However, our study has several limitations. First, although our specimens were fixed in the diastolic phase and the arterioles were maximally dilated, it is difficult to precisely reproduce physiologic intracoronary pressures when the postmortem specimens are injected with the Microfil ${ }^{\circledR}$. This inevitable difficulty may have some influence on 3D branching structures of arterioles and sometimes made a distinction between artery and vein difficult. Second, true branching generation is unknown in our arterioles because of the limited region sampled by the specimen "biopsy" and spatial resolution. Consequently, we evaluated the same size root arteriole and its downstream "tree" at all ages. Third, our image resolution is around $40 \mu \mathrm{m}$ because our images consist of $20-\mu \mathrm{m}$ cubic voxels. In addition, some technical obstacles such as air bubbles in the Microfil ${ }^{\circledR}$ within the microvessels might occur during the preparation of specimens, and this would result in "loss" of contrast within the lumen distal to that bubble. Consequently, lumen volumes and PVs would tend to be underestimated. Fourth, analysis of the vascular branching involved a small sample of all possible arteriolar beds in the specimens and analysis was focused on the main arteriole; hence the results may not adequately represent all arteriolar vasculature. Finally, the criterion used for detecting gray-scale thresholds (used to segment microvessels from the other tissue), combined with the decreased "brightness" of smaller branches due to image blurring, we selectively underestimated small-diameter arterioles.

Perfusion territory of the arterioles and its relation to growth. Several investigators have reported a relationship between the CSA of a coronary arterial segment and the volume of myocardial tissue that it perfuses (PV). The ratio PV/CSA in the LV walls of mature rats (14), dogs (15), and humans (16-18) has been reported as 1,500, 6,300, and $14,000 \mathrm{~mm}$, respectively. Our corresponding values in the combined LV wall and outflow tract for the three age groups are 356 (fetal), 934 (1 mo), and 3,269 (5 mo). The value for the 5-mo-old pig is consistent with those reported for other species, and the increase in value with age is consistent with a significant change in the myocardium and its metabolism during maturation, as well as reduced concentration of oxygen and glucose in fetal blood, which may require high cardiac output and/or ensure an oxygen margin of safety in the fetus (19). Although there was no difference in the PV/CSA ratio between the RV and LV in fetal and 1-mo-old pigs, the ratio was greater in the $\mathrm{LV}$ than that of the RV in 5-mo-old pigs. $\mathrm{LV}$ weight in rats has been reported to increase four times as fast as RV weight (20), and myocardial hypertrophy in the normal course of development in sheep has been reported to be much faster in LV than in RV in the early stage of extrauterine life (21). Along with a limited ability of an increasing number of arterioles after birth $(22,23)$, the rapid LV growth may result in larger perfusion territories.

We conclude from our results that the branching characteristics of vasculature in the $\mathrm{LV}$ and $\mathrm{RV}$ walls are the same and are consistent in general with branching characteristics of vasculature reported in the past (9). However, the results shown in Figure 5 indicate that values of the PV/CSA ratio in the LV are significantly higher than those in the RV. We speculate that this difference may be related to the reserve functional capacity that the LV is known to have, whereby its work load may vary considerably, much more so than that of the RV. It is also well-known that this reserve functional capacity of the LV is facilitated by a corresponding "coronary flow reserve" whereby coronary blood flow (primarily to the LV) may increase by as much as five- to sixfold (24). The results shown in Figure 5 also suggest that coronary flow
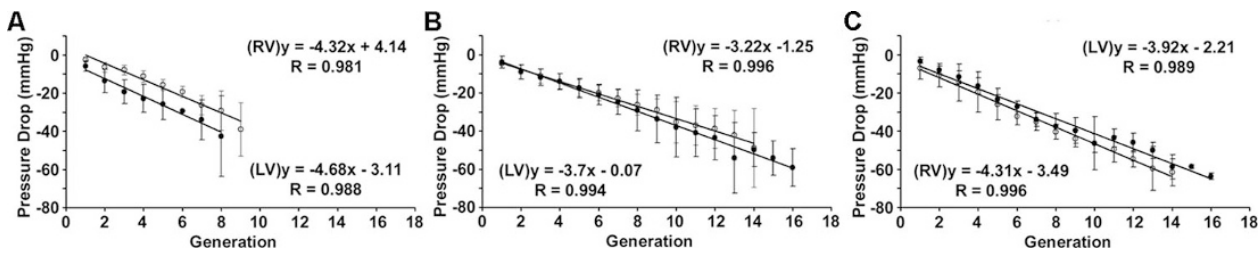

Figure 7. Normalized pressure decreases along the main arteriole in, $(A)$ fetal pig, (B) 1-mo-old pig, and (C) 5-mo-old old pig. Solid and open circles indicate LV and $\mathrm{RV}$, respectively. 
reserve to the LV is provided not by having vessels that have larger calibers than those in the RV but by having greater control over these calibers and hence over the resistance to flow in the LV. To the extent that the RV lacks this facility, it is less able to function under the more demanding conditions under which the LV must function.

In addition to clinical experiences $(1,2)$, the significant difference in coronary capillaries between congenitally and acquired pressure-overload LV walls strongly suggests that age and environmental factors have a strong impact on developing microvessels (25), and these observations may support our present conclusions, i.e. no initial structural difference in arterioles between RV and LV walls, followed by significantly greater perfusion territories within the mature LV wall. Therefore, investigation of arterioles in the pressure-overload RV wall at different stages of life may answer our initial question.

Acknowledgments. The authors thank Andrew J. Vercnocke and Diane R. Eaker for the micro-CT image reconstruction; Steven M. Jorgensen and David F. Hansen for the micro-CT scans; Dr. Jeffrey L. Platt, Department of Transplantation Biology, for providing the fetal and 1-mo-old pig hearts; Mara Lukenda and Delories C. Darling for editing and formatting the manuscript; and Dr. Tomoaki Ikeda, Department of Obstetrics and Gynecology at the National Cardiovascular Center, for informative suggestions.

\section{REFERENCES}

1. Murphy JH, Barlai-Kovach MM, Mathews RA, Beerman LB, Park SC, Neches WH, Zuberbuhler JR 1983 Rest and exercise right and left ventricular function late after the Mustard operation: assessment by radionuclide ventriculography. Am J Cardiol $51: 1520-1526$

2. Lubiszewska B, Gosiewska E, Hoffman P, Teresinska A, Rozanski J, Piotrowski W, Rydlewska-Sadowska W, Kubicka K, Ruzyllo W 2000 Myocardial perfusion and function of the systemic right ventricle in patients after atrial switch procedure for complete transposition: long-term follow-up. J Am Coll Cardiol 36:1365-1370

3. Singh TP, Humes RA, Muzik O, Kottamasu S, Karpawich PP, Di Carli MF 2001 Myocardial flow reserve in patients with a systemic right ventricle after atrial switch repair. J Am Coll Cardiol 37:2120-2125
4. Dimas AP, Moodie DS, Sterba R, Gill CC 1989 Long-term function of the morphologic right ventricle in adult patients with corrected transposition of the great arteries. Am Heart J 118:526-530

5. Kassab GS, Rider CA, Tang NJ, Fung YC 1993 Morphometry of pig coronary arterial trees. Am J Physiol 265:H350-H365

6. Fisher DJ, Heymann MA, Rudolph AM 1982 Regional myocardial blood flow and oxygen delivery in fetal newborn, and adult sheep. Am J Physiol 243:H729-H731

7. Goor DA, Lillehei CW 1975 Congenital Malformation of the Heart. Grune \& Stratton, New York, pp 11-15

8. Jorgensen SM, Demirkaya O, Ritman EL 1998 Three-dimensional imaging of vascular and parenchyma in intact rodent organs with x-ray micro-CT. Am J Physiol 275:H1103-H1114

9. Zamir M 1988 The branching structure of arterial trees. Comments Theor Biol $1: 15-37$

10. Hutchins GM, Bulkey BH, Miner MM, Boitnott JK 1977 Correlation of age and heart weight with tortuosity and caliber of normal human coronary arteries. Am Heart J 94:196-202

11. Murray CD 1926 The physiological principle of minimum work. I. The vascular system and the cost of blood volume. Proc Natl Acad Sci U S A 12:207-214

12. Zamir M 2000 The Physics of Pulsatile Flow. Springer-Verlag, New York, pp 1-220

13. Gossl M, Zamir M, Ritman EL 2004 Vasa vasorum growth in the coronary arteries of newborn pigs. Anat Embryol (Berl) 208:351-357

14. Lund PE, Naessens LC, Seaman CA, Reyes DA, Ritman EL 2000 Volume of myocardium perfused by coronary artery branches as estimated from 3D micro-CT images of rat heart. Proc SPIE Physiol Function Multidimensional Images 3978:314-319

15. Koiwa Y, Bahn RC, Ritman EL 1986 Regional myocardial volume perfused by the coronary artery branch: estimation in vivo. Circulation 74:157-163

16. O'Keefe JH, Owen RM, Bove AA 1987 Influence of left ventricular mass on coronary artery cross-sectional area. Am J Cardiol 59:1395-1397

17. Mela T, Meyer TE, Pape LA, Chung ES, Aurigemma GP, Weiner BH 1999 Coronary arterial dimension-to-left ventricular mass ratio in idiopathic dilated cardiomyopathy. Am J Cardiol 83:1277-1280

18. Seiler C, Kirkeeide RL, Gould KL 1992 Basic structure-function relation of the epicardial coronary vascular tree basis of quantitative coronary arteriography for diffuse coronary artery disease. Circulation 85:1987-2003

19. Richardson BS 1989 Fetal adaptive responses to asphyxia. Clin Perinatol 16:595611

20. Anversa P, Ricci R, Olivetti G 1986 Quantitative structural analysis of the myocardium during physiologic growth and induced cardiac hypertrophy: a review. J Am Coll Cardiol 7:1140-1149

21. Smolich JJ, Walker AM, Campbell GR, Adamson TM 1989 Left and right ventricular myocardial morphometry in fetal neonatal, and adult sheep. Am J Physiol 257:H1-H9

22. Rakusan K, Cicutti N, Flanagan MF 1994 Changes in the microvascular network during cardiac growth, development and aging. Cell Mol Biol Res 40:117-122

23. Heron MI, Kuo C, Rakusan K 1999 Arteriolar growth in the postnatal rat heart. Microvasc Res 58:183-186

24. Zamir M 2005 The Physics of Coronary Blood Flow. Springer, New York, pp $1-410$

25. Rakusan K, Flanagan MF, Geva T, Southern J, Van Praagh R 1992 Morphometry of human coronary capillaries during growth and the effect of age in left ventricular pressure-overload hypertrophy. Circulation 86:38-46 